\title{
Das SIWF-Fortbildungsdiplom: der «Goldstandard» in der Fortbildung
}

\author{
Christoph Hänggeli \\ Rechtsanwalt, Geschäftsführer des Schweizerischen Instituts für ärztliche Weiter- und Fortbildung SIWF
}

\section{Update Fortbildung}

Mit dem Ziel, den administrativen Aufwand für alle Beteiligten zu reduzieren, haben Plenum und Vorstand des SIWF beschlossen, die Fortbildungsplattform des Schweizerischen Instituts für ärztliche Weiter- und Fortbildung "kundenfreundlich» auszubauen. Die dafür notwendigen Arbeiten sind gegenwärtig im Gang: So wird beispielsweise ein zentrales Veranstaltungsregister die Suche nach den gewünschten Fortbildungsangeboten erleichtern, und der Eintrag der Veranstaltung ins Fortbildungsprotokoll wird automatisch durch den Organisator oder mittels Smartphone/ QR-Code erfolgen können.

Das Fortbildungsdiplom des SIWF wird zum "Goldstandard" als Nachweis für die Erfüllung der gesetzlichen Fortbildungspflicht. Wie in den umliegenden Ländern sollen auch in der Schweiz über $90 \%$ aller Ärztinnen und Ärzte in den Genuss eines gültigen Fortbildungsdiploms kommen. Die Ärzteschaft muss die ärztliche Fortbildung aus eigener Initiative glaubwürdig strukturieren und dokumentieren. Dies geht nicht ohne Qualitätssicherung und ohne eine minimale sinnvolle Kontrolle. Ansonsten läuft die Ärzteschaft Gefahr, dass ihre Anstrengungen von extern als zu wenig ernsthaft beurteilt werden und behördliche Interventionen zunehmend ihre Autonomie einschränken.

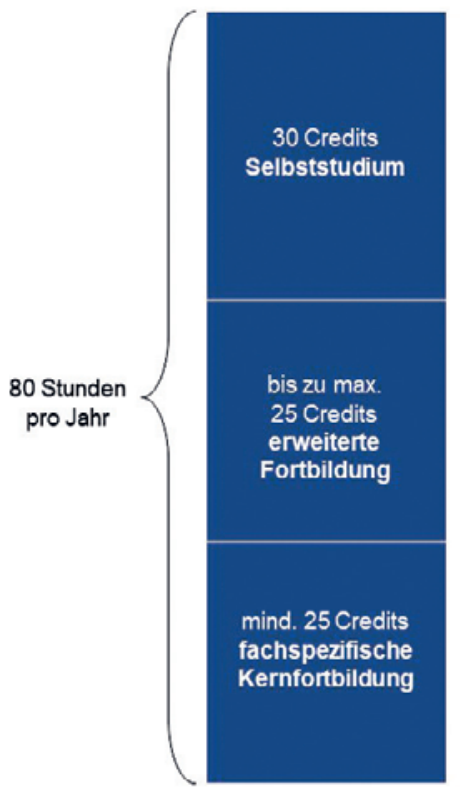

Nicht strukturierte Fortbildung

Nicht nachweispflichtig

Automatische Anrechnung

Strukturierte Fortbildung

Crediterteilung durch eine andere Fachgesellschaft (Facharztite) oder Schwerpunkt), eine kantonale Gesellschaft oder das SIWF. Im Bereich der Komplementärmedizin können auch folgende Gesellschaften Credits erteilen: ASA, VAOAS, SVHA, SANTH, SMGP.

Fachspezifische Kernfortbildung, welche die erforderlichen 25 Credits pro Jahr übersteigt

Nachweispflichtig

- Optional bis maximal 25 Credits anrechenbar

Abbildung 1: Gliederung der geforderten 80 Fortbildungsstunden pro Jahr.

- Anerkennung und Crediterteilung durch die Fachgesellschaft

Nachweispflichtig

Mindestens 25 Credits erforderlich

- Auflagen gemäss FBP der Fachgesellschaft

\section{Fortbildung: Selbstverständlichkeit und gesetzliche Berufspflicht}

Die Ärzteschaft versteht die Fortbildung als selbstverständliche Aufgabe, die primär eigenverantwortlich und entsprechend den individuellen Bedürfnissen wahrgenommen wird. Für alle fünf universitären Medizinalberufe ist sie im Medizinalberufegesetz (MedBG) als obligatorische Berufspflicht auch explizit vorgeschrieben. Die für die Kontrolle zuständigen kantonalen Gesundheitsbehörden sind befugt, eine Verletzung der Fortbildungspflicht mit einem Verweis oder einer Busse bis 20000 Franken zu ahnden. Die Ausgestaltung der gesetzlichen Fortbildungspflicht hingegen - d.h. insbesondere das Festlegen des Umfangs sowie der Art und Weise der Fortbildung - ist Sache der jeweiligen Berufsorganisation. Für die Ärzteschaft bietet das SIWF zusammen mit seinen Fachgesellschaften ein Fortbildungsdiplom an, das Gesundheitsbehörden und Krankenversicherer gleichermassen anerkennen. Der Erwerb des Fortbildungsdiploms ist laut Art. 9 der Fortbildungsordnung (FBO) obligatorisch. Für jeden Facharzttitel hat die zuständige Fachgesellschaft ein Fortbildungsprogramm erarbeitet, das die Voraussetzungen für den Erwerb des Diploms detailliert regelt. Welches der 45 Fortbildungsprogramme gewählt wird, liegt in der Eigenverantwortung jedes einzelnen Arztes.

Mit Vorteil orientieren sich Ärztinnen und Ärzte an demjenigen Fortbildungsprogramm, das der aktuellen Berufstätigkeit am ehesten entspricht. Die Fortbildungspflicht beginnt am 1. Januar nach dem Erwerb des Facharzttitels bzw. am 1. Januar nach der erstmaligen Aufnahme der ärztlichen Tätigkeit in der Schweiz. Wer sich hauptberuflich in Weiterbildung zu einem Facharzttitel oder Schwerpunkt befindet, ist während dieser Zeit nicht fortbildungspflichtig.

\section{Fachspezifische Kernfortbildung und erweiterte Fortbildung}

Als Richtmass für den Umfang der Fortbildung gelten 80 Stunden (= Credits) pro Jahr (Abb.1). Nachweispflichtig sind 50 Credits pro Jahr bzw. 150 Credits im Verlauf einer 


\section{Startselte Ärzte Fachgesellschaften Geschäftsstelle SIWF}

\section{Der einfache Weg zum Fortbildungsdiplom}

\section{\&}

Für Arzte

Hier können Sie ihre Fortbildungsaktivitäten protokollieren und Diplome beantragen.

\section{目}

\section{Für Fachgesellschaften}

Fachgesellschaften können hier die Diplomanträge fûr Ihr Fachgeblet bearbeiten.

Abbildung 2: Einstiegsseite zur Fortbildungsplattform.

\begin{tabular}{|c|c|c|}
\hline \multicolumn{2}{|c|}{ www.doctorfmh.ch - der FMH-ÄrzteIndex } & \multirow[b]{2}{*}{ E-Mail senden $\triangle$} \\
\hline Name, Vorname & von Hohenheim Theophrast & \\
\hline Mitglied der FMH & Ja & \\
\hline Arztdiplom(e) & 1972 & \\
\hline Facharzttitel & Psychiatrie und Psychotherapie, 2006 & \\
\hline $\begin{array}{l}\text { Fortbildungs-Diplom/- } \\
\text { Bestätigung }\end{array}$ & Psychiatrie und Psychotherapie (2020-2022) & \\
\hline Adresse(n) & $\begin{array}{l}\text { Praxis-Adresse } \\
\text { Praxis Paracelsus } \\
\text { Nussbaumstrasse } 29 \\
3000 \text { Bern } 15\end{array}$ & Lageplan $\rightarrow$ \\
\hline GLN-Nummer & 7600000000001 & \\
\hline Änderungen melden & $\begin{array}{l}\text { Ärztinnen und Ärzte können Änderungen zum } \\
\text { eigenen Eintrag direkt über myFMH erfassen. }\end{array}$ & myFMH $\rightarrow$ \\
\hline \multicolumn{3}{|c|}{ Informationen gemäss Angaben der Ärztin/des Arztes * } \\
\hline Akademischer Titel & Dr. med. & \\
\hline Sprachkenntnisse & Deutsch, Italienisch, Englisch & \\
\hline
\end{tabular}

Abbildung 3: Beispiel eines Eintrags auf www.doctorfmh.ch
Fortbildungsdiplome im Ärzteverzeichnis www.doctorfmh.ch

Das Ärzteverzeichnis www.doctorfmh.ch enthält neben allen offiziellen fachlichen Qualifikationen gemäss Weiterbildungsordnung (WBO) auch die Fortbildungsdiplome unter Angabe der Gültigkeitsperiode (Abb. 3). Weitere Informationen, wie zum Beispiel die im Protokoll erfassten Veranstaltungen, sind nicht ersichtlich. Der Datenschutz ist gewährleistet.

dreijährigen Fortbildungsperiode. Jedes Fortbildungsprogramm definiert eine fachspezifische Kernfortbildung im Umfang von 25 Credits pro Jahr. Hier kann die Fachgesellschaft verschiedene Fortbildungskategorien und deren maximale Anrechenbarkeit festlegen. Bis zu 25 Credits sind als "erweiterte Fortbildung» anrechenbar. Als erweiterte Fortbildung gelten alle Veranstaltungen, die von einer anderen Fachgesellschaft, von einer kantonalen Ärztegesellschaft oder vom SIWF Credits erhalten. Damit besteht die Möglichkeit, sich auch ausserhalb der eigenen Fachrichtung fortzubilden. Für Fragen zur Fortbildungspflicht in einem bestimmten Fachgebiet sind die jeweiligen Fachgesellschaften zuständig.

\section{Wie komme ich zum Fortbildungsdiplom?}

Ganz einfach: über die Fortbildungsplattform des SIWF (www.siwf.ch $\rightarrow$ Fortbildung $\rightarrow$ Fortbildungsplattform; Abb. 2). Hier erfassen Ärztinnen und Ärzte laufend ihre 


\section{Ausbau der Fortbildungsplattform}

Die Online-Fortbildungsplattform wird erweitert: Ein zentrales Fortbildungsregister soll die Suche nach den gewünschten Veranstaltungen erleichtern. Der Eintrag der Veranstaltung ins eigene Fortbildungsprotokoll erfolgt an der Veranstaltung mittels Smartphone/QR-Code oder durch den Veranstalter (automatisches Hochladen der Teilnahmebestätigung). Auch die Fachgesellschaften und Veranstalter profitieren: Sie können ihre Angebote auf der Plattform bekannt machen und zusätzliche Teilnehmer gewinnen. In einem ersten Schritt werden die nicht-fachspezifischen Fortbildungsveranstaltungen ins Register aufgenommen, für die das SIWF Credits erteilt. Das Plenum des SIWF hat die Finanzierung für die Entwicklung sichergestellt.

Fortbildungsaktivitäten in einem persönlichen Protokoll und beantragen das Diplom, sobald sie die geforderten 150 Credits gemäss dem gewählten Fortbildungsprogramm erreicht haben. Alle über die Fortbildungsplattform erworbenen Diplome erscheinen automatisch im offiziellen Ärzteverzeichnis www. doctorfmh.ch. Wer nicht über den entsprechenden Facharzttitel verfügt, erhält anstelle des Diploms eine Fortbildungsbestätigung zuhanden der Gesundheitsbehörden und Kostenträger.

\section{Ohne Fortbildungsdiplom keine Abrech- nung von Besitzstandleistungen!}

Besitzstandpositionen lassen sich weiterhin abrechnen, allerdings nur, sofern der Nachweis eines Fortbildungsdiploms erbracht ist. Zur Auswahl stehen die Fortbildungsprogramme der 45 Fachgesellschaften. Dank der erweiterten Fortbildung besteht in jedem Programm die Möglichkeit, sich in den Fachbereichen der gewünschten Besitzstandpositionen fortzubilden Die Besitzstandpositionen werden während der Gültigkeit des Fortbildungsdiploms entsprechend automatisch verlängert bzw. validiert.

\section{Fazit und Ausblick}

Nach der Weiterbildung ist die lebenslange Fortbildung für alle berufstätigen Ärztinnen und Ärzte eine Selbstverständlichkeit. Die Online-Fortbildungsplattform des SIWF und das Fortbildungsdiplom ermöglichen auf einfache Weise, die kompetente und dem aktuellen Stand der Medizin angepasste Berufsausübung nach aussen hin zu dokumentieren. Das Fortbildungsdiplom hat somit zwei Funktionen:

- Es erbringt den Nachweis für die gesetzliche Fortbildungspflicht, und

- es validiert alle Besitzstandpositionen.

\section{Fortbildung in 10 Punkten}

1. Die Fortbildungspflicht ist eine Berufspflicht, die in Art. 40 des MedBG verankert ist. Die Überwachung und Kontrolle obliegt den kantonalen Gesundheitsbehörden bzw. den kantonalen Ärztegesellschaften (bei entsprechender Delegation).

2. Das SIWF bietet zusammen mit den 45 Fachgesellschaften, die einen Facharzttitel repräsentieren, ein Fortbildungsdiplom an, das

- die Erfüllung der gesetzlichen Fortbildungspflicht gemäss MedBG bestätigt und

- die Besitzstandpositionen validiert.

3. Der Erwerb des Fortbildungsdiploms ist laut Art. 9 der Fortbildungsordnung (FBO) obligatorisch. Ärztinnen und Ärzte müs sen das Diplom mindestens in dem Fachgebiet erwerben, das ihrer hauptsächlichen aktuellen Berufstätigkeit entspricht.

4. Jedes Fortbildungsprogramm der 45 Fachgesellschaften unterscheidet zwischen folgenden Kategorien:

- Kernfortbildung (jährlich mindestens 25 Credits, kontrolliert)

- erweiterte Fortbildung (jährlich maximal 25 Credits, kontrolliert)

- Selbststudium (jährlich 30 Stunden, nicht kontrolliert) Die Fortbildungspflicht umfasst somit 80 Fortbildungsstunden pro Jahr. Alle drei Jahre sind 150 Credits nachzuweisen, wovon mindestens 75 Credits fachspezifische Kernfortbildung sein müssen
5. Wer TARMED-Besitzstandpositionen abrechnet, muss sich gemäss Dignitätskonzept auch in diesen Bereichen fortbilden. Die Fortbildung für Besitzstandpositionen lässt sich in der erweiterten Fortbildung mit 75 Credits pro drei Jahre absolvieren.

6. Das Fortbildungsdiplom ist alle drei Jahre zu erneuern.

7. Die in manchen Fähigkeitsausweisen vorgeschriebene Rezertifizierung ist unabhängig von den 45 Fortbildungsdiplomen der Facharzttitel geregelt. Das Nichtbeachten der Rezertifizierungsregeln kann zum Verlust des Ausweises führen. Dasselbe gilt für interdisziplinäre Schwerpunkte.

8. Weitere Informationen über die 45 Fortbildungsprogramme und die Ansprechpersonen der jeweiligen Fachgesellschaften sind zu finden auf www.siwf.ch in der Rubrik Fortbildung. Zu beachten sind insbesondere die FAQs.

9. Über die Fortbildungsplattform (www.siwf.ch $\rightarrow$ Fortbildung $\rightarrow$ Fortbildungsplattform) lassen sich die Fortbildungsaktivitäten laufend erfassen, und nach Erreichen der geforderten 150 Credits kann das Fortbildungsdiplom nach Genehmigung durch die Fachgesellschaft selbst ausgedruckt werden.

10. Für das Corona-Jahr 2020 haben alle fortbildungspflichtigen Ärztinnen und Ärzte automatisch eine "Gutschrift Covid-19" im Umfang von 35 Credits in ihrem Fortbildungsprotokoll erhalten. 